\title{
Review of: "The Differences in Distant Metastatic Patterns and Their Corresponding Survival Between Thyroid Cancer Subtypes"
}

\author{
Rasa Zarnegar ${ }^{1}$ \\ 1 Cornell University
}

Potential competing interests: The author(s) declared that no potential competing interests exist.

I think its an interesting paper but is limited in its data.

I think the authors need to focus on each cancer type and define disease specific mortality and cause. le was death from metastatic foci or from the local invasion. This is especially important for ATC were loco regional invasion is most likely the cause of mortality. Therefore specific KM curves are required comparing single or multifocal metastasis.

I think patient selection is also critical here and a control group. Also when looking at MTC the calcitonin and CEA are required.

For PTC the role and dose of RAI and mutation status is need.

I think the paper is lumping too many diseases into the same and I dont this that maybe prudent. 